\title{
Impairment of serum albumin antioxidant properties in obstructive sleep apnoea syndrome
}

\author{
P. Faure*,\#, R. Tamisier, , J-P. Baguet ${ }^{+}$, A. Favier*, S. Halimi ${ }^{\S}$, P. Lévy ${ }^{\#, q}$ and \\ J-L. Pépin $\#$,
}

ABSTRACT: Antioxidant counteraction of oxidative stress has been poorly explored in obstructive sleep apnoea (OSA). Serum albumin is a major antioxidant agent and structural modifications induced by glucose or free radicals impair its antioxidant properties.

The aim of the present study was to compare antioxidant capacities and structural changes of albumin in nonobese OSA patients and healthy volunteers.

Albumin structural changes were studied by quenching of fluorescence in the presence of acrylamide. Albumin thiols and fructosamines, reflecting oxidation- and glycation-induced changes in serum albumin, respectively, were assessed. Albumin structural changes were demonstrated by a significant decrease in quenching of fluorescence in OSA patients. Oxidation, resulting in a significant decrease in thiol groups (3.7 \pm 0.7 versus $2.3 \pm 0.4 \mu \mathrm{mol} \cdot \mathrm{g}^{-1}$ protein), and glycation, associated with a significant increase in fructosamines $(226.6 \pm 27$ versus $\left.286 \pm 44.4 \mu \mathrm{mol} \cdot \mathrm{L}^{-1}\right)$, were found when comparing healthy volunteers with OSA patients. There was a significant relationship between both parameters and sleep apnoea severity. After continuous positive airway pressure intervention, albumin thiol groups were reassessed in seven of the 16 OSA patients and increased significantly from $2.25 \pm 0.39$ to $2.79 \pm 0.31 \mu \mathrm{mol} \cdot \mathrm{g}^{-1}$ protein.

Obstructive sleep apnoea patients demonstrated a reduction in serum albumin antioxidant properties that may aggravate oxidative stress and, thus, contribute to cardiovascular and metabolic morbidities.

KEYWORDS: Albumin, antioxidant properties, obstructive sleep apnoea, oxidative stress, protein glycation, thiols

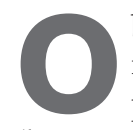

bstructive sleep apnoea (OSA) syndrome is characterised by recurrent episodes of partial or complete pharyngeal collapse (hypopnoeas or apnoeas) occurring during sleep. It is a growing health concern affecting up to $5 \%$ of middle-aged males and females in the general population [1]. This is a serious health hazard, being recognised as an independent risk factor for hypertension, arrhythmias and coronary heart disease $[2,3]$. People with OSA have a night-time peak in occurrence of sudden death, and an increased rate of cardiovascular morbidity and mortality $[2,3]$.

The desaturation-reoxygenation sequence is a typical pattern coupled with the majority of respiratory events. This sequence leads to oxidative stress with production of reactive oxygen species (ROS) [4]. Numerous studies have demonstrated increased oxidative stress in OSA using various biological markers, although comorbidities, such as diabetes, hypertension or obesity, may account for part of these results [47]. The increased levels of ROS contribute to the generation of adhesion molecules [8], activation of leukocytes [9] and production of systemic inflammation [10]. In combination, these mechanisms generate vascular endothelium damage. Moreover, high sympathetic output, as consistently found in OSA, may lead to insulin resistance even in nonobese OSA patients [11], thus representing an additional source of oxidative stress.

Oxidative stress is characterised by an imbalance between the production and degradation of ROS. While numerous studies have addressed the issue of increased ROS production, only a limited number of studies have addressed the role of antioxidant capacities in OSA patients. BARCELÓ et al. [12] reported an alteration in antioxidant capacities, with a reduction in total antioxidant
AFFILIATIONS

*Dept of Integrated Biology (DBI)

'Sleep and Exercise Laboratory (EFCR),

${ }^{+}$Dept of Cardiology and Hypertension,

${ }^{\S}$ Endocrinologie, Diabétologie, Nutrition, DigiDUNE, University Hospital Grenoble, and \#HP2 Laboratory (Hypoxia Pathophysiology) INSERM ERI 17 Joseph Fourier University, Grenoble, France.

CORRESPONDENCE

J-L. Pépin

EFCR et Laboratoire du sommeil

CHU de Grenoble

BP 217

38043 Grenoble Cedex 09

France

Fax: 33476765586

E-mail: JPepin@chu-grenoble.fr

Received:

May 242007

Accepted after revision:

January 222008

SUPPORT STATEMENT

This work was supported by grants from the Comité des Maladies Respiratoires de l'Isère (COMARES), La Tronche (Grenoble, France); the scientific council of AGIR à dom, Meylan (Grenoble); and the Délégation Régionale la Recherche Clinique (DRRC) of the University Hospital Grenoble (Grenoble)

STATEMENT OF INTEREST None declared. 
status (TAS) and a decrease in vitamin A and vitamin E levels in OSA patients. In the same study, continuous positive airway pressure (CPAP) treatment normalised TAS [12].

Albumin represents the most abundant protein in the circulatory system with a significant antioxidant activity, which is principally related to the protein's capacity to bind metal ions and scavenge free radicals [13-15]. It has been demonstrated that, besides its plasma concentration, structural modifications induced by glucose or free radicals impaired the antioxidant properties of albumin. The current hypothesis was that antioxidant defences mediated by albumin might be altered in OSA patients, as exposure to oxidative stress and insulin resistance are both parts of the disease.

In order to test this hypothesis, both the antioxidant capacity and the structural changes of serum albumin were investigated in selected OSA patients with a body mass index (BMI) $\leqslant 30 \mathrm{~kg} \cdot \mathrm{m}^{-2}$ and in healthy volunteers serving as a control group.

\section{METHODS}

\section{Study population}

In total, 23 subjects were studied: seven healthy volunteers and 16 OSA patients. The healthy volunteers were recruited from the staff of the University Hospital Grenoble (Grenoble, France). Patients were recruited according to the presence of OSA syndrome and a BMI $\leqslant 30 \mathrm{~kg} \cdot \mathrm{m}^{-2}$. Patients were nondiabetic, nonsmoking, lean subjects. They were free of cardiovascular symptoms and medications. Cardiovascular examinations, including blood pressure measurements and ECG, were normal. Current smoking was an exclusion criterion as it would potentially induce oxidative stress. Ethical approval was obtained from the local ethics committee at the University Hospital Grenoble (Grenoble). All of the participants gave written informed consent. All participants underwent sleep studies and an assessment of different biological parameters as follows.

\section{Sleep studies}

Polysomnographic recordings were performed and scored following standard criteria [16]. Airflow was measured via nasal pressure, associated with the sum of the buccal and nasal thermistor signals. Respiratory efforts were monitored with abdominal and thoracic bands. An additional signal of respiratory effort (i.e. pulse transit time) was recorded concurrently. Arterial oxygen saturation $\left(\mathrm{Sa}_{1} \mathrm{O}_{2}\right)$ was measured using a pulse oximeter (Biox-Ohmeda 3700 ; Ohmeda, Liberty Corner, NJ, USA). An apnoea was defined as a complete cessation of airflow for $\geqslant 10 \mathrm{~s}$. A hypopnoea was a reduction of $\geqslant 50 \%$ in the nasal pressure signal for $\geqslant 10 \mathrm{~s}$, or a decrease of $30-50 \%$ in the nasal pressure signal for $\geqslant 10 \mathrm{~s}$ combined with either oxygen desaturation of $\geqslant 3 \%$ or an electroencephalogram (EEG) arousal (defined according to the American Academy of Sleep Medicine report) [16]. Apnoeas were classified as obstructive, central or mixed according to the presence or absence of respiratory efforts. The classification of hypopnoeas as obstructive or central was based upon the pulse transit time signal and the shape of the inspiratory part of nasal pressure (flow-limited aspect or not). An apnoea/hypopnoea index (AHI) was calculated and defined as the number of apnoeas and hypopnoeas per hour of sleep (full polysomnography) or per hour of recording (polysomnography without EEG recording). Sleep apnoea was defined as an AHI $\geqslant 15$ events $\cdot h^{-1}$.

\section{Biological parameters}

Metabolic parameters

Glucose, triglycerides, total cholesterol, high- and low-density lipoprotein (HDL and LDL, respectively) cholesterol were measured enzymatically on a Modular 700 automated analyser (Roche Diagnostics, Meylan, France). Insulin was measured using a radio-immunometric sandwich assay (CIS Bio International, Gif-sur-Yvette, France) with two monoclonal antibodies. The homeostasis model assessment (HOMA) was calculated using the following formula:

$$
\begin{aligned}
& \mathrm{HOMA}=\left(\text { fasting serum insulin }\left(\mu \text { units } \cdot \mathrm{mL}^{-1}\right) \times\right. \text { fasting } \\
& \text { blood glucose } \left.\left(\mathrm{mmol} \cdot \mathrm{L}^{-1}\right)\right) / 22.5
\end{aligned}
$$

None of the present study patients exhibited diabetes, defined as fasting venous plasma glucose level $\geqslant 7.0 \mathrm{mmol} \cdot \mathrm{L}^{-1}$.

Oxidative stress characterisation: urinary 8-isoprostaglandin F2 $\alpha$ type III assays

Urinary 8-isoprostaglandin (iP)F2 $\alpha$ type III measurements were performed by enzyme immunoassay to provide a marker of oxidative stress. Urine samples were kept refrigerated during the collection period, after which they were transferred to the laboratory, aliquoted and stored at $-20^{\circ} \mathrm{C}$. They were extracted on a solid phase extraction workstation using ISOLUTE C18 (EC) cartridges at $100 \mathrm{mg} / 10 \mathrm{~mL}$ (International Sorbent Technology, Hengoed, UK). Solid phase eluates of urine samples were tested using validated enzyme immunoassays. Polyclonal antiserum for iPF2 $\alpha$-III, and standard and acetylcholinesterase tracers were provided from Cayman Chemical (Ann Arbor, MI, USA). The assays were derived from a standard competition immunoassay procedure using microtitre plates pre-coated with mouse antirabbit immunoglobulin G (96-well plates).

\section{Albumin structural changes, glycation and oxidation}

After isolating albumin from human plasma, its structural changes, glycation and oxidation were studied (fig. 1). A schematic representation of the glycation- and oxidationinduced changes in the structure and properties of human serum albumin (HSA) are shown in figure 1, adapted from BOURDON et al. [17].

\section{Isolation of albumin from human plasma}

Blue Sepharose ${ }^{\mathrm{TM}} 6$ Fast Flow (GE Healthcare Bio-Sciences AB, Uppsala, Sweden) was used to purify plasma albumin. The gel was first washed with $50 \mathrm{mM}$ Tris $\mathrm{HCl}$ buffer ( $\mathrm{pH} 7.4$ ) containing $0.5 \mathrm{M} \mathrm{NaCl}$, and packed into a column of $1.5 \mathrm{~cm}$ diameter, $4 \mathrm{~cm}$ height. The column was equilibrated with five bed volumes of $50 \mathrm{mM}$ Tris $\mathrm{HCl}, 0.5 \mathrm{M} \mathrm{NaCl}$ buffer. The gel was then transferred into $5-\mathrm{mL}$ tubes and the technique of batch separation was used to obtain solutions of purified and concentrated albumin from plasma. Briefly, plasma $(1.35 \mathrm{~mL})$ was applied to the gel $(2.5 \mathrm{~mL})$. After the first centrifugation $\left(4,000 \times g\right.$ for $10 \mathrm{~min}$ at $\left.4{ }^{\circ} \mathrm{C}\right)$, the supernatant was eliminated. The gel was washed three times with the starting buffer, in the same conditions. The gel-bound albumin was eluted in $650 \mu \mathrm{L}$ with $50 \mathrm{mM}$ Tris $\mathrm{HCl}$ buffer ( $\mathrm{pH} 7.4$ ) containing $1.5 \mathrm{M} \mathrm{NaCl}$. The tightly bound proteins were removed with $0.5 \mathrm{M} \mathrm{NaSCN}$ 

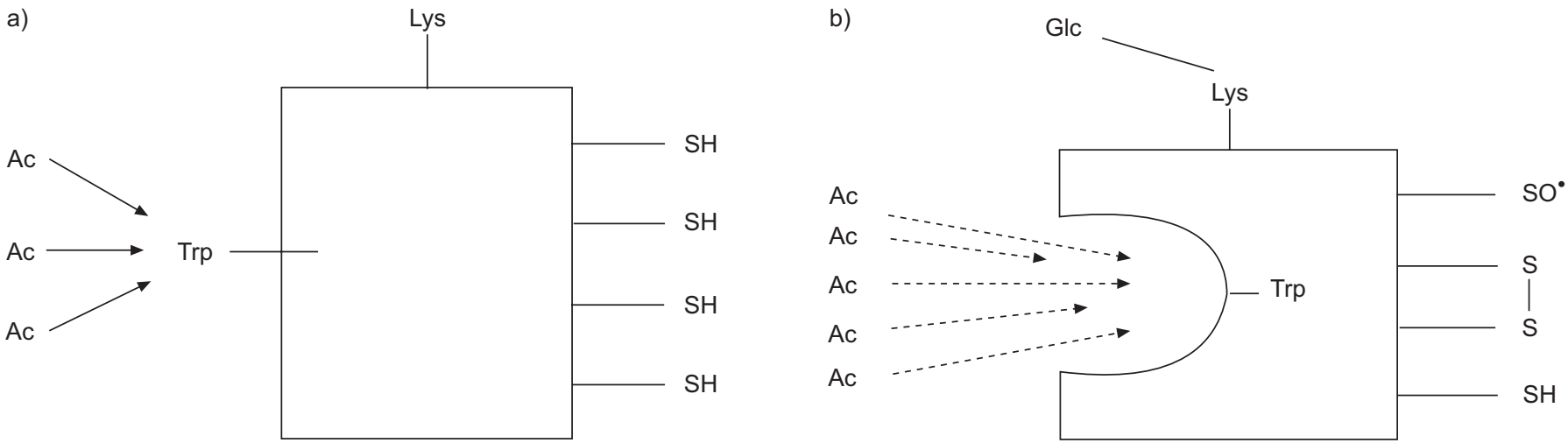

FIGURE 1. Schematic models of the glycation- and oxidation-induced changes in the structure and properties of human serum albumin (HSA). a) HSA in its normal globular conformation presents an important intrinsic fluorescence due to its tryptophan residues (Trp). The quenching of fluorescence with acrylamide (Ac) was used to investigate the structural changes of HSA. The antioxidant activity of HSA is due to the free radical-trapping property of thiol groups in their reduced form (SH). Therefore, HSA thiols were measured. Lysine residues (Lys) are in their free form. Fructosamines were measured as a marker of albumin glycation on Lys residues. b) The hypothesis of the present study was that albumin changes would be associated with sleep apnoea. The conformation of free radical-stimulated HSA is greatly altered, with a markedly modified conformation as assessed by a marked decrease in Trp fluorescence. More Ac is then needed to obtain the same level of fluorescence. There are numerous glycated (Glc) Lys residues (expressed as an increase in fructosamines). Thiols are oxidised to form both disulphur linkages $(\mathrm{S}-\mathrm{S})$ and thiyl radicals $\left(\mathrm{SO}^{\circ}\right)$. The number of thiol groups in reduced form (SH) is decreased. Adapted from [17].

and the gel was re-equilibrated with three bed volumes of starting buffer.

\section{Quenching of fluorescence}

The albumin intrinsic fluorescence and quenching of fluorescence in the presence of acrylamide could be used [17] since HSA contains two tryptophan residues (Trp 218 and Trp 134), which generate autofluorescence. Moreover, it was previously shown that acrylamide does not alter the protein conformation [17]. Fluorescence measurements were performed using an LS 50 fluorimeter (PerkinElmer, Courtaboeuf, France) at excitation and emission wavelengths of 278 and $343 \mathrm{~nm}$, respectively. Acrylamide was used as a quencher agent and was added to albumin solution in final concentration of $20 \mathrm{~g} \cdot \mathrm{L}^{-1}$ in $0.5 \mathrm{M}$ phosphate buffer ( $\mathrm{pH} 7.4$ ), to a final concentration up to $60 \mathrm{mM}$ acrylamide. The results are expressed as mean values of I0/I (Stern-Volmer constant, $\mathrm{Kq}$ ), where I0 was albumin fluorescence before adding the quencher and I was the albumin fluorescence after adding the quencher.

\section{Measurement of albumin thiol groups}

HSA contains one free cysteine-derived, redox-reactive thiol group (Cys 34). Due to its reactive properties, the Cys 34 thiol moiety confers to HSA a major role in serum antioxidant capacity. Albumin thiols account for $80 \%\left(500 \mu \mathrm{mol} \cdot \mathrm{L}^{-1}\right)$ of the thiols in plasma [18].

The measurement of thiol groups was performed using Ellman's reagent. Briefly, $2.5 \mathrm{mM}$ 5,5'-dithiobis(2-nitrobenzoic acid) in $0.2 \mathrm{M}$ phosphate buffer, $\mathrm{pH} 8.0$, was mixed with $500 \mu \mathrm{L}$ of sample and $750 \mu \mathrm{L}$ of $50 \mathrm{mM}$ phosphate buffer, $\mathrm{pH} \mathrm{8.0,} \mathrm{and}$ baseline absorbance was recorded at $412 \mathrm{~nm}$. Following this, $250 \mu \mathrm{L}$ of freshly prepared Ellman's reagent was added, the reaction was allowed to proceed for $15 \mathrm{~min}$ at room temperature in the dark, and the final absorbance was measured. HSA thiol group values were expressed in $\mu \mathrm{mol} \cdot \mathrm{g}^{-1}$ protein using a molar absorption coefficient of $13,600 \mathrm{~L} \cdot \mathrm{mol}^{-1} \cdot \mathrm{cm}^{-1}$ for thiol 5,5'-dithiobis(2-nitrobenzoic acid) complex. A calibration curve was performed by sequential dilution of a $1 \mathrm{mM} \mathrm{N}$-acetylcysteine stock solution.

Albumin thiol groups were reassessed after $15 \pm 4$ months of CPAP intervention in seven of the 16 OSA patients.

Measurement of plasma fructosamines

Plasma fructosamines were measured on a Modular 700 automated analyser (Roche Diagnostics).

\section{Statistical analysis}

Sample size power calculation

In a previous study [14], type 2 diabetic patients showed a $0.7 \pm 0.48$ difference in thiol groups between patients and controls. As the present OSA patients were supposed to be only suffering from insulin resistance, a mean difference of 0.6 with the same SD was expected. A total of seven healthy volunteers and 15 OSA patients provided a power of 0.80 with a value of $\alpha=0.05$.

The normality of the data distribution was assessed. Continuous data were expressed as mean \pm SD. Relationships between the continuous variables were evaluated by Pearson's or Spearman's correlation analysis when data were not normally distributed. Differences in average for the SternVolmer constants between the two groups and according to the different acrylamide concentrations were determined by twofactor repeated measure ANOVA. Comparisons of two continuous variables were made with unpaired t-tests, except when comparing OSA patients before and after CPAP treatment, when paired t-tests were used. The MannWhitney U-test was used when the data were not normally distributed. Both univariate and multivariate analysis were performed, in order to assess the respective contribution of different factors in explaining the variance in albumin thiols and fructosamines. Multivariate analysis included all the variables potentially influencing the studied variable: AHI, 
TABLE 1 Anthropometric, sleep and metabolic parameters

\begin{tabular}{|c|c|c|c|}
\hline Characteristics & HV & OSA & p-value \\
\hline Subjects $n$ & 7 & 16 & \\
\hline BMI $\mathbf{k g} \cdot \mathrm{m}^{-2}$ & $23.8 \pm 1.9$ & $26.8 \pm 2.8$ & $<0.05$ \\
\hline Fasting glucose $\mathrm{mmol} \cdot \mathrm{L}^{-1}$ & $4.6 \pm 0.2$ & $4.9 \pm 0.3$ & $<0.01$ \\
\hline HOMA & $0.9 \pm 0.8$ & $1.9 \pm 1.3$ & $<0.05$ \\
\hline LDL & $1.1 \pm 0.3$ & $1.5 \pm 0.3$ & NS \\
\hline $\mathrm{HDL}$ & $0.6 \pm 0.2$ & $0.5 \pm 0.1$ & NS \\
\hline Triglycerides $\mathbf{g} \cdot \mathrm{L}^{-1}$ & $1.2 \pm 0.6$ & $1.4 \pm 0.6$ & NS \\
\hline Serum albumin $\mathrm{g} \cdot \mathrm{L}^{-1}$ & $43.6 \pm 2.4$ & $43.1 \pm 2.9$ & NS \\
\hline Urinary isoprostaglandins $\mathrm{mg} \cdot \mathrm{nmol}^{-1}$ of creatinine & $7.7 \pm 3$ & $13.6 \pm 11$ & 0.14 \\
\hline $\mathrm{Sa}, \mathrm{O}_{2}$ mean \% & $95.1 \pm 1$ & $93.5 \pm 2.2$ & 0.05 \\
\hline $\mathrm{Sa}_{\mathrm{a}, \mathrm{O}_{2}} \min \%$ & $88.7 \pm 2.3$ & $83.5 \pm 7.5$ & $<0.05$ \\
\hline Time $^{\#} \mathrm{Sa}, \mathrm{O}_{2}<\mathbf{9 0 \%}$ & $0.1 \pm 1.3$ & $9.8 \pm 19.4$ & NS \\
\hline hsCRP $\mathrm{mg} \cdot \mathrm{L}^{-1}$ & $0.6 \pm 0.2$ & $2.6 \pm 2.6$ & $<0.05$ \\
\hline
\end{tabular}

Data are presented as mean $\pm \mathrm{SD}$, unless otherwise stated. HV: healthy volunteers; OSA: obstructive sleep apnoea; BMI: body mass index; HOMA: homeostasis model assessment; LDL: low-density lipoprotein; HDL: high-density lipoprotein; $\mathrm{AHI}$ : apnoea/hypopnoea index; $\mathrm{Sa}, \mathrm{O}_{2}$ : arterial oxygen saturation; hsCRP: high-sensitivity Creactive protein; NS: nonsignificant. ${ }^{\#}$ : percentage of total sleep time.

mean nocturnal $\mathrm{Sa}_{2} \mathrm{O}_{2}$, minimal nocturnal $\mathrm{Sa}_{2} \mathrm{O}_{2}$, fasting glucose, HOMA index, clinical blood pressure, BMI, cholesterol and Creactive protein (CRP). Variables significantly related with the

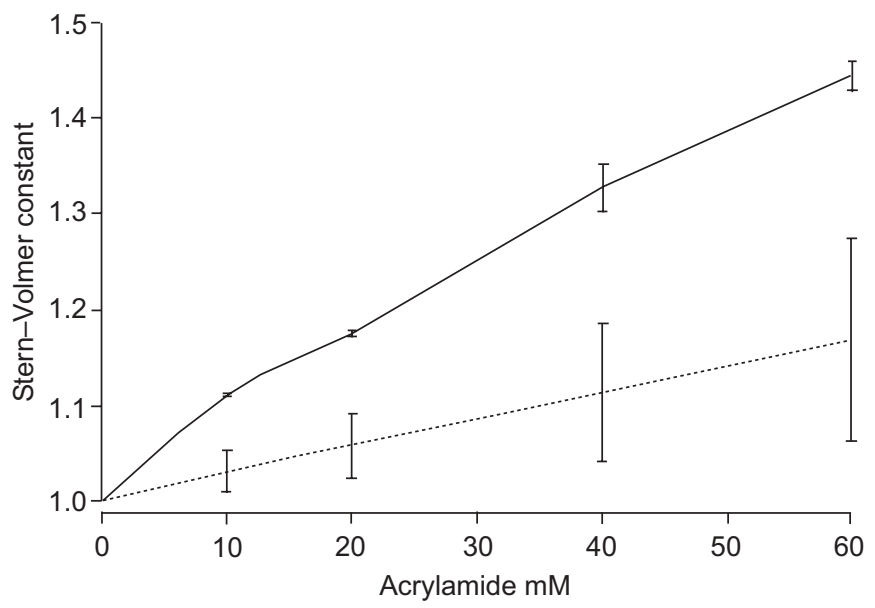

FIGURE 2. Albumin structural changes: quenching of fluorescence in the presence of acrylamide. The Stern-Volmer curves, representing tryptophan accessibility to the quencher, were significantly different for the albumin preparations tested from healthy volunteers (-) compared with obstructive sleep apnoea (OSA) patients $(\cdots ; p<0.001$ ANOVA). Moreover, the increase in SternVolmer constant with acrylamide concentration was different in healthy volunteers versus OSA patients $(p<0.001$ ANOVA). This shows that both global accessibility to tryptophan and magnitude of response decreased in OSA patients owing to albumin structural changes. Error bars represent SD. studied variable in univariate analysis were finally taken into account for the model. Values of $\mathrm{p}<0.05$ were considered significant.

\section{RESULTS}

\section{Anthropometric, sleep and metabolic parameters}

Healthy volunteers $(n=7)$ and OSA patients $(n=16)$ were similar in age but did show differences in BMI (table 1). Patients had an average BMI value $<27 \mathrm{~kg} \cdot \mathrm{m}^{-2}$ but significantly higher than healthy volunteers. Compared with healthy volunteers, OSA patients exhibited higher fasting glucose and HOMA index values. Patients exhibited higher total and LDL cholesterol values, and lower HDL cholesterol. These differences reached significance only for total cholesterol values. By design, data from sleep analysis differed between healthy volunteers and OSA patients. High-sensitivity (hs)CRP levels were significantly higher in the whole group of OSA patients in comparison with healthy volunteers $(2.6 \pm 2.6$ versus $\left.0.6 \pm 0.2 \mathrm{mg} \cdot \mathrm{L}^{-1} ; \mathrm{p}<0.01\right)$. Clinical blood pressure, even in the normal range, was higher in OSA patients compared with controls. Serum albumin concentration was not different between the OSA patients and the control group $(43.13 \pm 2.87$ and $43.57 \pm 2.37 \mathrm{~g} \cdot \mathrm{L}^{-1}$, respectively).

\section{Oxidative stress characterisation: urinary iPF2 $\alpha-$ III assays}

In OSA patients, urinary iPF2 $\alpha$-III values were two times the average values of control subjects, although this did not reach statistical significance $\left(13.6 \pm 11\right.$ versus $7.7 \pm 3 \mathrm{mg} \cdot \mathrm{nmol}^{-1}$ of creatinine, $p=0.14)$. There was no significant correlation 

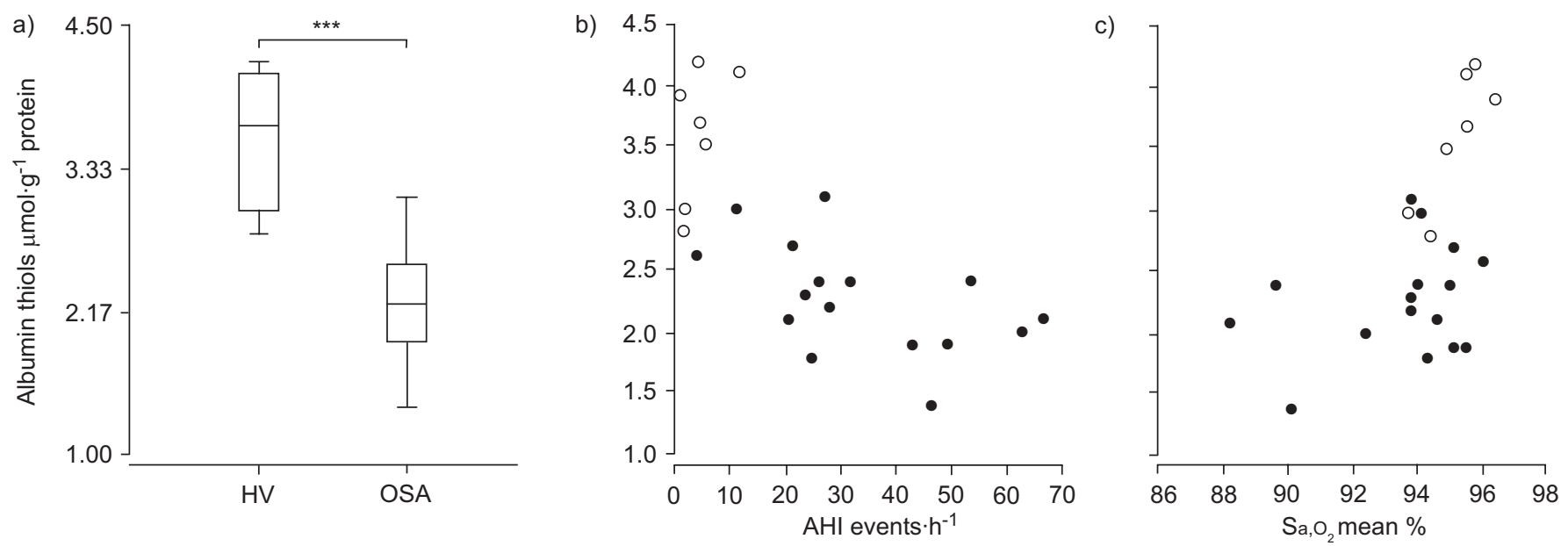

FIGURE 3. Albumin oxidation evaluated by analysis of albumin thiol groups. a) Obstructive sleep apnoea (OSA) patients showed a significant decrease in albumin thio groups compared with healthy volunteers (HV). Box-and-whisker plots presenting the first, second and third quartiles (box) and maximum and minimum values (whiskers). Significant correlations were found between b) albumin thiols and apnoea/hypopnoea index ( $\mathrm{AHI})$ and c) albumin thiols and mean nocturnal arterial oxygen saturation $\left(\mathrm{Sa}, \mathrm{O}_{2}\right)$ O: HV; • : OSA patients. ${ }^{* * *: ~} \mathrm{p}<0.001$.

between this measurement of oxidative stress and changes detected in serum albumin.

\section{HSA structural changes, oxidation and glycation}

Structural changes as assessed by quenching of fluorescence in the presence of acrylamide

Fluorescence of HSA could be quenched by increasing concentrations of acrylamide (fig. 2). The quasi-linearity of the plots indicated that the quenching process followed a collision type. The Stern-Volmer curves, representing tryptophan accessibility to the quencher, were significantly different for the different albumin preparations tested when comparing healthy volunteers and OSA patients, $(p<0.001$, ANOVA). The magnitude of the increase in Stern-Volmer constant with higher acrylamide concentrations was blunted in OSA patients compared with healthy volunteers ( $p<0.001$, ANOVA).
Oxidation of HSA evaluated by analysis of albumin thiol groups OSA patients exhibited a significant decrease in albumin thiol groups compared with healthy volunteers $(2.3 \pm 0.4$ versus $3.7 \pm 0.7 \mu \mathrm{mol} \cdot \mathrm{g}^{-1}$ protein, respectively; $\mathrm{p}<0.001$; fig. $\left.3 \mathrm{a}\right)$. There was a significant relationship between albumin thiols and AHI $(\mathrm{r}=-0.71, \mathrm{p}<0.001$; fig. $3 \mathrm{~b})$ and albumin thiols and mean nocturnal $\mathrm{Sa}_{1} \mathrm{O}_{2}(\mathrm{r}=0.45, \mathrm{p}<0.05$; fig. $3 \mathrm{c})$. There was a significant negative correlation between thiol groups and HOMA index $(\mathrm{r}=-0.47, \mathrm{p}<0.02)$.

HSA glycation as measured by fructosamine levels

There was a significant increase in fructosamines when comparing healthy volunteers and OSA patients $(226.6 \pm 27$ versus $286 \pm 44.4 \mu \mathrm{mol} \cdot \mathrm{L}^{-1}$, respectively; $\mathrm{p}<0.001$; fig. $\left.4 \mathrm{a}\right)$. Significant correlations were found between fructosamines and AHI $(r=0.62, p<0.001$; fig. $4 b)$ and between fructosamines
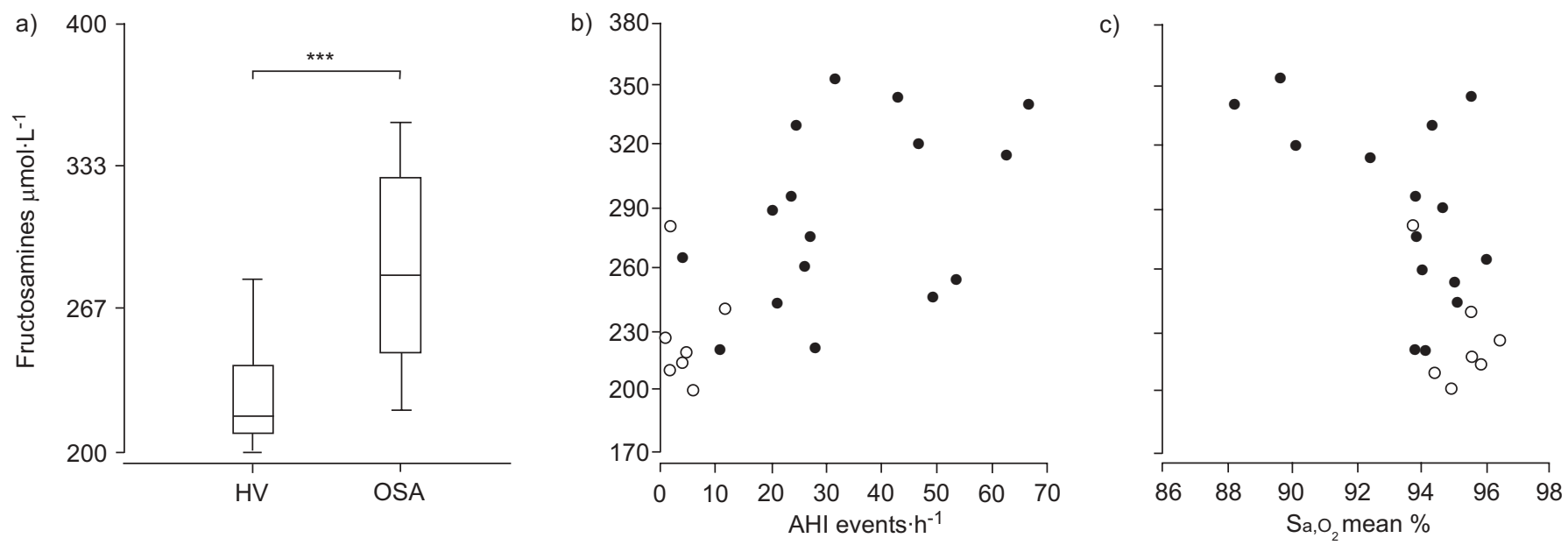

FIGURE 4. Albumin glycation measured by fructosamine levels. a) Obstructive sleep apnoea (OSA) patients showed a significant increase in fructosamines compared with healthy volunteers (HV). Box-and-whisker plots presenting the first, second and third quartiles (box) and maximum and minimum values (whiskers). Significant correlations were found between b) fructosamines and apnoea/hypopnoea index (AHI) and c) fructosamines and mean nocturnal arterial oxygen saturation $\left(\mathrm{Sa}, \mathrm{O}_{2}\right)$. $\mathrm{O}$ : $\mathrm{HV}$;

- : OSA patients. ${ }^{* *}: \mathrm{p}<0.001$. 

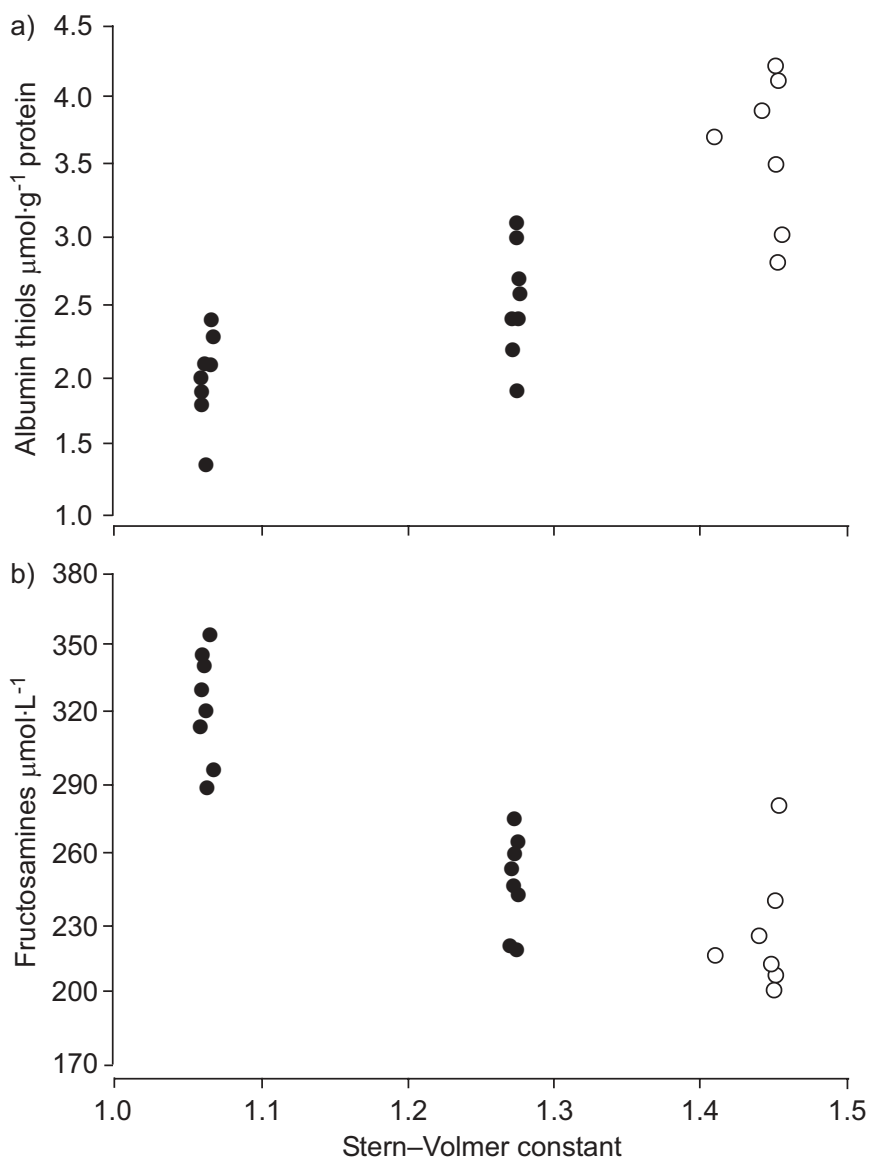

FIGURE 5. Albumin structural changes were related to the intensity of albumin oxidation and glycation. Stern-Volmer constants at $60 \mathrm{mM}$ acrylamide, reflecting albumin structural changes, were closely related to the parameters of a) albumin oxidation as measured by albumin thiols and b) glycation as measured by fructosamines. $\bigcirc$ : healthy volunteers; $\mathbf{0}$ : obstructive sleep apnoea patients. a) $r=0.85, p<0.001$; b) $r=-0.77, p<0.001$

and mean nocturnal $\mathrm{Sa}_{1} \mathrm{O}_{2}(\mathrm{r}=-0.52, \mathrm{p}<0.01$; fig. $4 \mathrm{c})$. There was also a significant correlation between fructosamines and HOMA index $(\mathrm{r}=0.52, \mathrm{p}<0.01)$.

There were close correlations between Stern-Volmer constants and both albumin oxidation and glycation (albumin thiols: $\mathrm{r}=0.85, \mathrm{p}<0.001$; fructosamines: $\mathrm{r}=-0.77, \mathrm{p}<0.001$; fig. 5).

\section{Multivariate analysis}

Albumin thiols

Univariate linear regression demonstrated that AHI, BMI, HOMA index and systolic blood pressure contributed to 50, 34, 29 and $17 \%$ of albumin thiols variance, respectively. Multivariate analysis showed that $\mathrm{AHI}$ had an independent effect on albumin thiols $(p<0.04)$. Conversely, BMI was not an independent factor $(\mathrm{p}=0.37)$.

\section{Plasma fructosamines}

Univariate linear regression demonstrated that AHI, BMI and HOMA index contributed to 39,33 and $59 \%$ of albumin thiols variance, respectively. Multivariate regression analysis showed that only the HOMA index had an independent effect

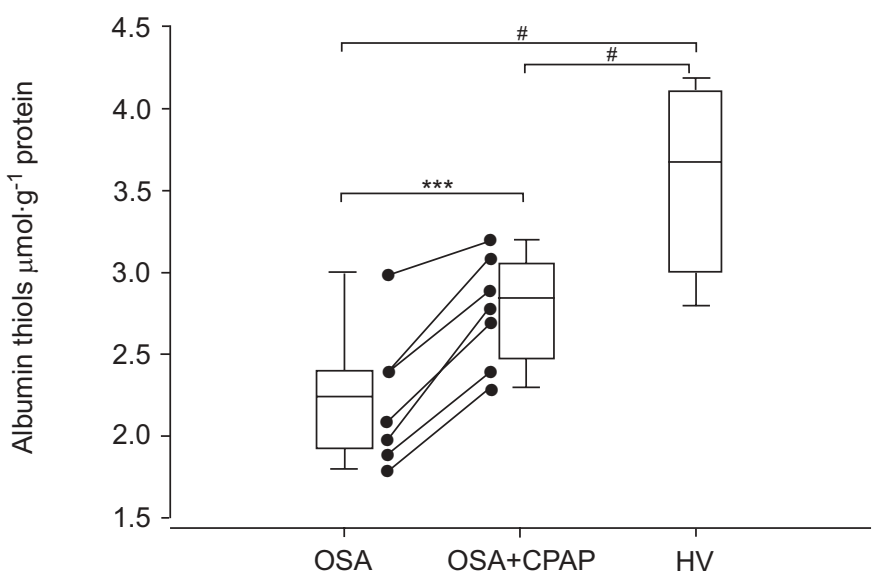

FIGURE 6. Albumin thiol groups before and after continuous positive airway pressure (CPAP) treatment in obstructive sleep apnoea (OSA) patients. Individual values (-), as well as box-and-whisker plots showing first, second and third quartiles and maximum and minimum values. $\mathrm{HV}$ : healthy volunteers. ${ }^{*}: \mathrm{p} \leqslant 0.01$; $\star * *: p<0.001$

on plasma fructosamines $(\mathrm{p}=0.01)$, while AHI was not an independent factor.

\section{Effects of CPAP intervention}

After a mean of $15 \pm 4$ months of CPAP intervention, albumin thiol groups were reassessed in seven of the 16 OSA patients, and had increased significantly from $2.25 \pm 0.39$ to $2.79 \pm 0.31 \mu \mathrm{mol} \cdot \mathrm{g}^{-1}$ protein $(\mathrm{p}<0.001$; fig. 6$)$.

\section{DISCUSSION}

For the first time, a decrease in serum albumin antioxidant capacities has been demonstrated in nonobese OSA patients, when compared with healthy volunteers. This impaired antioxidant capacity was related to the severity of OSA as expressed both by $\mathrm{AHI}$ and the amount of nocturnal oxygen desaturation. Moreover, CPAP intervention significantly increased albumin thiol groups.

Quenching of fluorescence in the presence of acrylamide provided arguments for albumin structural changes. As more acrylamide was needed in apnoeic patients compared with healthy volunteers to obtain a given level of fluorescence, this demonstrated that tryptophan accessibility on serum albumin molecules was decreased in OSA. This reduced accessibility was explained by serum albumin structural changes occurring both via protein oxidation (decrease in thiols) and protein glycation (increase in fructosamines). It has been demonstrated in vitro that protein heating induces identical structural changes, closely associated with a progressive loss of antioxidant properties [15]. More specifically, negative correlations have been established between the number and availability of thiol groups and albumin antioxidant properties [15].

The three main mechanisms linking sleep apnoea to cardiovascular diseases are: imbalance between ROS production and degradation [4], a source of oxidative stress; metabolic dysfunction as expressed by insulin resistance [11]; and chronic systemic inflammation [10]. The changes in albumin 
properties reported in the present study may be involved in all three mechanisms.

As HSA mainly works as a free radical scavenger [19], the reduction in antioxidant activity shown in OSA in the present study leads to an increase in oxidative stress. In OSA, oxidative stress is related to OSA per se and also to cardiovascular and metabolic comorbidities [7]. As the study patients were free of such comorbidities, there was only a trend towards an increase in isoprostaglandins compared with controls, and no direct correlation was found between oxidative stress and albumin structural changes. However, the present results are in line with the previous demonstration of a negative correlation between OSA severity and antioxidant capacity as measured by TAS $[12,20]$. TAS is a global measure of serum antioxidant properties, including different pathways; thus, one can hypothesise that changes in albumin antioxidant capacities are part of the explanation for TAS reduction.

In the present study, OSA subjects were free from diabetes but demonstrated altered insulin sensitivity, with a mean HOMA value significantly higher than controls. It is well known that insulin resistance is linked with oxidative stress and associated with reduced intracellular antioxidant defences [21]. In the present study, there was a significant relationship between the HOMA index and albumin oxidation as indicated by the decrease in thiol groups $(\mathrm{r}=-0.47, \mathrm{p}<0.02)$. In addition, the HOMA index was also associated with fructosamine increase $(\mathrm{r}=-0.52, \mathrm{p}<0.01)$. Elevated fructosamine levels, reflecting glycation of albumin, are recognised in diabetes mellitus as being associated with impairment in antioxidant capacity. The same type of effect is likely to be present in OSA. Glycated albumin has been demonstrated to be associated with cardiovascular changes, both in animal models [22] and in human diabetes [23-25]. It has also been shown in mice that endothelial cell damage caused by glycated albumin can be reversed by monoclonal antibodies specific to glycated albumin [22]. In diabetic patients, an increase in glycated albumin level has been reported to be associated with an increase in pulse pressure, a well studied marker of cardiovascular risk [23]. Glycated albumin has also been shown to be correlated with plasma markers of endothelial dysfunction and cell adhesion molecules [24], both of which have already been described in OSA as potential major risk factors for cardiovascular morbidity [8, 26]. Glycated HSA is also able to increase proliferation as well as migration of vascular smooth muscle cells [25]. Additionally, these intermediate glycated products may lead to the formation of advanced glycation end products (AGEs). It has been recently demonstrated that AGE serum levels are increased in nondiabetic OSA patients, and are associated with the severity of the disease [27]. As the most abundant plasma protein exhibiting significant glycation, as demonstrated in the present study, albumin might contribute to AGE formation in severe OSA patients.

OSA patients also suffer from vascular inflammation. In the present study, hsCRP was significantly increased in OSA patients compared with healthy volunteers. In an in vitro cell model submitted to intermittent hypoxia, as well as in apnoeic patients, RYAN and co-workers [28, 29] demonstrated an increase in the pro-inflammatory cytokines tumour necrosis factor- $\alpha$ and interleukin (IL)- 6 , mediated by selective activation of the pro-inflammatory transcription factor nuclear factor (NF)-кB. Interestingly, albumin augments intracellular glutathione levels in lymphocytes and modulates activation of $\mathrm{NF}-\kappa \mathrm{B}$ [30]. A thiol-mediated reduction in serum albumin decreases glutathione levels, thus allowing NF- $\kappa \mathrm{B}$ activation [30]. Glycated serum albumin also induces NF- $\kappa$ B activation in vascular smooth muscle cells, increases activity of p38 mitogen-activated protein (MAP) kinases leading to increased production of monocyte chemotactic protein (MCP)-1 and IL-6. Increased production of MCP-1 and IL- 6 has also been reported in sleep apnoea [8]. Moreover, using an endothelial cell in vitro model, it has been shown that intermittent hypoxia activates p38 MAP kinases and pharmacological inhibition of p38 MAP kinases abolishes NF- $\mathrm{kB}$ activation [29].

In conclusion, the present study demonstrated for the first time a decrease in albumin antioxidant properties in severe nonobese obstructive sleep apnoea patients. This was related to albumin oxidation and glycation, and appeared to be correlated with the severity of obstructive sleep apnoea and also with insulin sensitivity impairment. Moreover, this was reversible under effective therapy by continuous positive airway pressure. These changes contribute to an unfavourable balance between oxidative stress and antioxidant capacities and may also contribute to aggravation of systemic inflammation.

\section{ACKNOWLEDGEMENTS}

The authors would like to thank N. Arnol for statistical analysis and M. Peeters for clinical research management, both from the Sleep and Exercise Laboratory (EFCR), University Hospital Grenoble (Grenoble, France).

\section{REFERENCES}

1 Young T, Peppard PE, Gottlieb DJ. Epidemiology of obstructive sleep apnea: a population health perspective. Am J Respir Crit Care Med 2002; 165: 1217-1239.

2 Gami AS, Howard DE, Olson EJ, Somers VK. Day-night pattern of sudden death in obstructive sleep apnea. $N$ Engl J Med 2005; 352: 1206-1214.

3 Marin JM, Carrizo SJ, Vicente E, Agusti AG. Long-term cardiovascular outcomes in men with obstructive sleep apnoea-hypopnoea with or without treatment with continuous positive airway pressure: an observational study. Lancet 2005; 365: 1046-1053.

4 Lavie L. Obstructive sleep apnoea syndrome-an oxidative stress disorder. Sleep Med Rev 2003; 7: 35-51.

5 Lavie L, Vishnevsky A, Lavie P. Evidence for lipid peroxidation in obstructive sleep apnea. Sleep 2004; 27: 123-128.

6 Barceló A, Miralles C, Barbé F, Vila M, Pons S, Agustí AG. Abnormal lipid peroxidation in patients with sleep apnoea. Eur Respir J 2000; 16: 644-647.

7 Svatikova A, Wolk R, Lerman LO, et al. Oxidative stress in obstructive sleep apnoea. Eur Heart J 2005; 26: 2435-2439.

8 Ohga E, Tomita T, Wada H, Yamamoto H, Nagase T, Ouchi Y. Effects of obstructive sleep apnea on circulating ICAM-1, IL-8, and MCP-1. J Appl Physiol 2003; 94: 179-184.

9 Schulz R, Mahmoudi S, Hattar K, et al. Enhanced release of superoxide from polymorphonuclear neutrophils in obstructive sleep apnea. Impact of continuous positive 
airway pressure therapy. Am J Respir Crit Care Med 2000; 162: 566-570.

10 Ciftci TU, Kokturk O, Bukan N, Bilgihan A. The relationship between serum cytokine levels with obesity and obstructive sleep apnea syndrome. Cytokine 2004; 28: 87-91.

11 Punjabi NM, Ahmed MM, Polotsky VY, Beamer BA, O'Donnell CP. Sleep-disordered breathing, glucose intolerance, and insulin resistance. Respir Physiol Neurobiol 2003; 136: 167-178.

12 Barceló A, Barbé F, de la Peña M, et al. Antioxidant status in patients with sleep apnoea and impact of continuous positive airway pressure treatment. Eur Respir J 2006; 27: 756-760.

13 Bourdon E, Blache D. The importance of proteins in defense against oxidation. Antioxid Redox Signal 2001; 3: 293-311.

14 Faure $\mathrm{P}$, Troncy L, Lecomte $\mathrm{M}$, et al. Albumin antioxidant capacity is modified by methylglyoxal. Diabetes Metab 2005; 31: 169-177.

15 Rondeau P, Armenta S, Caillens H, Chesne S, Bourdon E. Assessment of temperature effects on $\beta$-aggregation of native and glycated albumin by FTIR spectroscopy and PAGE: relations between structural changes and antioxidant properties. Arch Biochem Biophys 2007; 460: 141-150.

16 Sleep-related breathing disorders in adults: recommendations for syndrome definition and measurement techniques in clinical research. The Report of an American Academy of Sleep Medicine Task Force. Sleep 1999; 22: 667689.

17 Bourdon E, Loreau N, Blache D. Glucose and free radicals impair the antioxidant properties of serum albumin. FASEB J 1999; 13: 233-244.

18 Quinlan GJ, Martin GS, Evans TW. Albumin: biochemical properties and therapeutic potential. Hepatology 2005; 41: 1211-1219.

19 Bourdon E, Loreau N, Lagrost L, Blache D. Differential effects of cysteine and methionine residues in the antioxidant activity of human serum albumin. Free Radic Res 2005; 39: 15-20.
20 Christou K, Moulas AN, Pastaka C, Gourgoulianis KI Antioxidant capacity in obstructive sleep apnea patients. Sleep Med 2003; 4: 225-228.

21 Ceriello A, Motz E. Is oxidative stress the pathogenic mechanism underlying insulin resistance, diabetes, and cardiovascular disease? The common soil hypothesis revisited. Arterioscler Thromb Vasc Biol 2004; 24: 816-823.

22 Cohen MP, Clements RS, Cohen JA, Shearman CW. Glycated albumin promotes a generalized vasculopathy in the $\mathrm{db} / \mathrm{db}$ mouse. Biochem Biophys Res Commun 1996; 218: 72-75.

23 Schram MT, Schalkwijk CG, Bootsma AH, et al. Advanced glycation end products are associated with pulse pressure in type 1 diabetes: the EURODIAB Prospective Complications Study. Hypertension 2005; 46: 232-237.

24 Schalkwijk CG, Ligtvoet N, Twaalfhoven H, et al. Amadori albumin in type 1 diabetic patients: correlation with markers of endothelial function, association with diabetic nephropathy, and localization in retinal capillaries. Diabetes 1999; 48: 2446-2453.

25 Hattori Y, Suzuki M, Hattori S, Kasai K. Vascular smooth muscle cell activation by glycated albumin (Amadori adducts). Hypertension 2002; 39: 22-28.

26 Ip MS, Tse HF, Lam B, Tsang KW, Lam WK. Endothelial function in obstructive sleep apnea and response to treatment. Am J Respir Crit Care Med 2004; 169: 348-353.

27 Tan KC, Chow WS, Lam JC, et al. Advanced glycation endproducts in nondiabetic patients with obstructive sleep apnea. Sleep 2006; 29: 329-333.

28 Ryan S, Taylor CT, McNicholas WT. Selective activation of inflammatory pathways by intermittent hypoxia in obstructive sleep apnea syndrome. Circulation 2005; 112: 2660-2667.

29 Ryan S, McNicholas WT, Taylor CT. A critical role for p38 MAP kinase in NF- $\kappa \mathrm{B}$ signaling during intermittent hypoxia/reoxygenation. Biochem Biophys Res Commun 2007; 355: 728-733.

30 Cantin AM, Paquette B, Richter M, Larivée P. Albuminmediated regulation of cellular glutathione and nuclear factor $\kappa \mathrm{B}$ activation. Am J Respir Crit Care Med 2000; 162: 1539-1546. 